\title{
Cardiovascular Screening and Lipid Management in Breast Cancer Survivors
}

\author{
S. Tucker Price, MD, PhD, Lisa D. Mims, MD, Marty S. Player, MD, MSCR, \\ Carole Berini, MS, Suzanne Perkins, MPH, Chanita Hughes Halbert, PhD, and \\ Vanessa A. Diaz, MD, MSCR
}

Background: Cardiovascular disease (CVD) is the leading cause of death among breast cancer (BC) survivors. BC survivors are at increased risk of CVD due to a higher prevalence of risk factors. Current data are limited on the cardiovascular screening practices and lipid management in this population in primary care settings.

Methods: A retrospective case control study was performed with $105 \mathrm{BC}$ survivors and 210 matched controls (based on age and medical comorbidities of diabetes, hypertension, and hyperlipidemia). BC survivors were established with primary care practices within a large academic institution and had completed primary cancer treatment. Data on screening for CVD and lipid management were collected via a retrospective chart review.

Results: The average BC survivor was 63 years old, with 9 years since diagnosis. Compared with matched controls, BC survivors had more cholesterol screening $(88 \%$ vs $70 \%, P<.001)$ and active statin prescriptions $(63 \%$ vs $40 \%, P<.05)$ if indicated by the Atherosclerotic Cardiovascular Disease Calculator. There were no differences in CVD screening in White and African American BC survivors. However, African American BC survivors were more likely to have hypertension $(P<.01)$ and have a body mass index in the overweight and obese category $(P<.001)$ than White BC survivors. Older BC survivors were more likely to receive cholesterol screening.

Discussion: This study demonstrates that BC survivors who have an established primary care provider have improved cholesterol screening and statin therapy based on their risk of developing chronic diseases. (J Am Board Fam Med 2020;33:894-902.)

Keywords: Cardiovascular Diseases, Case-Control Studies, Hyperlipidemias, Obesity, Prevalence, Retrospective Studies, Risk Factors, Statins, Survivorship

\section{Introduction}

In 2016, there were over 3.5 million women living in the United States with a history of breast cancer (BC). ${ }^{1}$ Cardiovascular disease (CVD) is the most common cause of death in older BC survivors. ${ }^{2,3}$

This article was externally peer reviewed.

Submitted 19 December 2019; revised 19 May 2020; accepted 20 May 2020.

From the Department of Family Medicine, Medical University of South Carolina, Charleston, SC (STP, LDM, MSP, CB, SP, VAD); Medical University of South Carolina Department of Psychiatry and Behavioral Sciences, Charleston, SC (CHH).

Funding: This project was supported in part by the National Center for Advancing Translational Sciences of the National Institutes of Health under Grant Number UL1 TR001450. The content is solely the responsibility of the authors and does not necessarily represent the official views of the National Institutes of Health.

Conflict of interest: None.

Corresponding author: Sarah Tucker Price, MD, PhD, Medical University of South Carolina, 135 Cannon Street, Suite
Furthermore, BC survivors are at increased risk of CVD when compared with individuals without history of cancer. $^{4-11}$ The etiology of CVD in BC survivors is multifactorial. $\mathrm{BC}$ and $\mathrm{CVD}$ share common risk factors, including activation of inflammatory pathways. ${ }^{12-16}$ Management of comorbid medical conditions has also been shown to be suboptimal during active cancer treatment, and cancer disease processes themselves may cause subclinical myocardial damage. ${ }^{2}$ Cancer therapies, including radiotherapy, chemotherapy, and endocrine therapies, can negatively impact cardiovascular health in 3 domains: vascular conditions, structural problems, and cardiac dysfunction. ${ }^{17-23}$ The risk of CVD increases with

405, MSC \#192 Charleston, SC 29425-0192 (E-mail: marrison@musc.edu). 
additional risk factors and exposures, as the "multihit hypothesis" has proposed. ${ }^{2,22}$

There are limited guidelines for screening for CVD in BC survivors; however, studies are ongoing regarding the use of biomarkers, imaging modalities, and preventive medications for cardiovascular risk reduction. ${ }^{2,23-25}$ Current strategies for risk factor reduction in $\mathrm{BC}$ survivors include aggressive management of comorbid conditions, including hypertension, diabetes, hyperlipidemia, and promotion of lifestyle factors such as smoking cessation, maintaining an appropriate body weight, and increased physical activity. ${ }^{3,19,23,26-28}$

BC survivors are followed by multiple specialists, which often includes oncology, primary care, and cardiology. Communication between primary care and oncology is often insufficient with poor differentiation of roles in the care of cancer survivors. ${ }^{29-35}$ Cancer survivors who see both cancer specialists and primary care received a wider range of appropriate clinical services as compared with those seeing cancer specialists alone. ${ }^{36-39}$

Limited studies have evaluated cardiovascular screening practices in BC survivors and have focused predominantly on those over the age of 65 years, an age at which there is decreased benefit to preventive interventions. BC survivors self report increased cholesterol screening compared with the general population, although claims-based data demonstrate decreased receipt of preventive health services and screenings, including cholesterol screening. ${ }^{16,38}$ Empirical data are not available on the type of provider who is likely to screen BC survivors for CVD risk. Chidwick et $\mathrm{al}^{40}$ demonstrated similar initiation of statin therapy in BC survivors with high cardiovascular risk as compared with a control population. However, when evaluating statin use 1-year following initiation, cancer survivors had increased rates of discontinuation.

Despite increased risk of CVD and cardiovascular mortality for BC survivors compared with the general population, cardiovascular screening practices and risk reduction through statin therapy has not been evaluated and has, in particular, not been evaluated to our knowledge in a cohort that includes individuals under the age of 65 years. Investigation has also not been conducted regarding the specialty providing this care. In this study, we sought to evaluate cholesterol screening and management in BC survivors compared in a retrospective case-control study with further analysis regarding differences between African American and White BC survivors.

\section{Methods \\ Study Design}

A retrospective case-control study with 105 BC survivors and 210 matched controls was conducted at the Medical University of South Carolina (MUSC). The project received MUSC Institutional Review Board approval with a waiver of informed consent. Inclusion criteria for BC survivors were individuals between 40 and 75 years old with previous diagnosis of BC not currently receiving active cancer therapy. Endocrine therapy (ie, aromatase inhibitors, tamoxifen, ovarian suppression) was not considered active therapy for the purpose of this study. BC survivors included had identified MUSC Primary Care as their primary care provider with a primary care visit between December 2017 and February 2018. There are 20 outpatient clinics that comprise MUSC Primary Care, composed of internal medicine (26\%) and family medicine (51\%) physicians and advanced practice providers (23\%). Exclusion criteria included individuals who were currently receiving active primary treatment or hospice care. History of CVD was not considered an exclusion criterion; however, disease incidence is documented. Cancer diagnosis outside of superficial skin cancer was not found in cases or controls. BC survivors were matched with individuals without history of malignancy seen at the same primary care clinics within 3 months at a 2:1 ratio of controls to cases to improve the power of the study. ${ }^{41}$ Matching criteria included age and the presence or absence of the medical comorbidities of diabetes, hypertension, and hyperlipidemia. These comorbidities were selected based on their impact on screening parameters and statin use. An electronic medical record inquiry was conducted among individuals seen by the associated primary care clinics with ICD codes consistent with diagnosis of $\mathrm{BC}$. A random number generator was used to assign a number to each of the eligible individuals identified. The first 105 individuals study criteria were included to meet the sample size established through power analysis. A list of control individuals was generated, when greater than 2 individuals met matching criteria each match was assigned a random number and those receiving the lowest 2 numbers were selected. In 5 cases, a match meeting the criteria of age and comorbidities was unavailable so a match consistent with at least 1 comorbidity was used.

\section{Data Collection}

Data extraction was initially conducted by a research data warehouse inquiry and then subsequently by 
manual chart review for the data unable to be extracted through the initial query. Demographic data obtained included age and race/ethnicity. Clinic variables obtained included date of last primary care provider visit. Cancer-related variables for cases included year of diagnosis, stage, treatments received, and receptor status. Comorbidity data included the presence or absence of hypertension, hyperlipidemia, diabetes, myocardial infarction, cerebrovascular accident, and coronary artery disease. Outcome variables included last blood pressure, body mass index, date and ordering provider of last lipid panel, statin therapy and ordering provider, atherosclerotic cardiovascular disease (ASCVD) risk, and hemoglobin A1c. Chart review was conducted by the principal investigator and subsequently verified in a population of $10 \%$ of randomly selected participants by our data analyst for manually extracted data with a Cohen's kappa value of 0.933 for inter-rater reliability. Individuals with a diagnosis of hypertension were determined to have controlled blood pressure if their most recent blood pressure reading was $<140 / 90$ based on the Eighth Joint National Committee recommendations. ${ }^{42}$ An individual was considered to have received screening for hyperlipidemia if a lipid panel or its component pieces had been obtained within 3 years. ASCVD risk was calculated for each individual (ages 40 to 75 years based on inclusion criteria) without history of diabetes, coronary artery disease, or known cardiovascular event based on the most recent blood pressure and lipid panel. ${ }^{43}$ Indications for statin therapy included a 10 -year ASCVD greater than $7.5 \%$, current diagnosis of diabetes, or previous major cardiovascular event which includes myocardial infarction or cerebrovascular accident. All individuals with documented coronary artery disease on their problem list had a statin indication based on the criteria above. The specialty of the ordering provider was determined by review of the electronic medical record of the ordering provider for the test or medication. For the purposes of this study, potentially cardiotoxic therapies received included radiation therapy, anthracyclines, endocrine therapies, and trastazumab.

\section{Statistical Considerations}

A power calculation was conducted to determine the sample size needed to detect a $15 \%$ difference in cholesterol screening with $\alpha$ of 0.05 and $\beta 0.85$ and an enrollment ratio of 2:1. IBM SPSS v25 was used for statistical analyses. Descriptive statistics were calculated for all variables. Group means and standard deviations are presented for continuous variable. Counts and percentages are presented for categorical values. Comparisons were made between groups using the Student's t-test and Pearson's c2 analysis as applicable. $P<.05$ was considered statistically significant for all analyses. Binary logistic regression was conducted for lipid screening and statin use for cancer survivors and controls and based on race with use of categories of White as compared with other races which was predominantly African American.

\section{Results \\ Study Population}

The average age of $\mathrm{BC}$ survivors was 63 years, with an average time of diagnosis 9.4 years prior and median time of diagnosis 6 years prior. The racial distribution of individuals included in the study as well as the incidence of medical comorbidities in shown in Table 1. Clinical information related to BC for the study sample is provided in Table 1. Of the BC survivors, $6.7 \%$ were found to have had a previous heart attack or stroke, compared with $1.4 \%$ of controls $(P<.05)$. Minor differences in the incidence of hyperlipidemia and diabetes were found due to limitations in matching all participants with an equivalent age and comorbidity matched control. ASCVD risk was calculated for those individuals for whom it was appropriate to calculate and is shown in Table 1. Body mass index was the same between BC survivors and matched controls. Primary care provided $93.7 \%$ of the cholesterol screening and prescribed $93.6 \%$ of statins to BC survivors (Table 1). Cardiology was the next most common specialty providing cholesterol screening and statin therapy.

\section{Cardiovascular Screening and Lipid Management in BC Survivors}

$\mathrm{BC}$ survivors were more likely than controls to receive cholesterol screening $(P<.001)$ and statin therapy $(P<.05)$ than age and comorbidity matched controls (Figure 1A). There was no significant difference in blood pressure control in cancer survivors and controls or the percentage of individuals who were overweight or obese based on body mass index (Figure 1A). No significant differences between cardiovascular screening and lipid management were observed based on time of diagnosis with $\mathrm{BC}$, stage at time of diagnosis, or treatments received (data not shown). When blood pressure control was assessed 
Table 1. Demographic Data

\begin{tabular}{|c|c|c|}
\hline & $\begin{array}{c}\mathrm{BC} \\
\text { Survivors }\end{array}$ & $\begin{array}{l}\text { Matched } \\
\text { Control }\end{array}$ \\
\hline Age, mean (SD) & $63(8.5)$ & $63(8.5)$ \\
\hline Individuals, $\mathrm{n}$ & 105 & 210 \\
\hline \multicolumn{3}{|l|}{ Ethnicity } \\
\hline White & $48 \%(51)$ & $57 \%(120)$ \\
\hline African American & $44 \%(46)$ & $39 \%(82)$ \\
\hline Hispanic or Latino & $4 \%(4)$ & $1 \%(2)$ \\
\hline Asian & $4 \%(4)$ & $3 \%(6)$ \\
\hline \multicolumn{3}{|l|}{ Medical comorbidities } \\
\hline Hypertension & $63 \%(66)$ & $63 \%(132)$ \\
\hline Hyperlipidemia & $32 \%(34)$ & $34 \%(73)$ \\
\hline Diabetes & $27 \%(28)$ & $29 \%(61)$ \\
\hline \multicolumn{3}{|l|}{ Cardiotoxic therapies received } \\
\hline None & $25 \%(26)$ & \\
\hline One or more & $77 \%(81)$ & \\
\hline Two or more & $42 \%(44)$ & \\
\hline \multicolumn{3}{|l|}{ Cancer therapy } \\
\hline Radiation therapy & $38 \%(40)$ & \\
\hline Chemotherapy & $38 \%(40)$ & \\
\hline Endocrine therapy & $55 \%(58)$ & \\
\hline \multicolumn{3}{|l|}{ Stage at diagnosis } \\
\hline 0 & $16 \%(17)$ & \\
\hline 1 & $36 \%(38)$ & \\
\hline 2 & $22 \%(23)$ & \\
\hline 3 & $4 \%(4)$ & \\
\hline 4 & $2 \%(2)$ & \\
\hline Unknown & $20 \%(21)$ & \\
\hline \multicolumn{3}{|l|}{ Cardiovascular metrics } \\
\hline ASCVD risk & $10.9 \%(8.6)$ & $11.9 \%(8.1)$ \\
\hline Body mass index & $30.4(7.6)$ & $30.9(9.9)$ \\
\hline Major cardiovascular events & $6.7 \%(7)$ & $1.4 \%(3)$ \\
\hline $\begin{array}{l}\text { Hemoglobin A1c <9\% } \\
(\mathrm{n}=89)\end{array}$ & $67.2 \%(20)$ & $71.4 \%(41)$ \\
\hline $\begin{array}{l}\text { Lipid screening by primary } \\
\text { care }\end{array}$ & $93.7 \%(90)$ & $88.3 \%(129)$ \\
\hline $\begin{array}{l}\text { Statin therapy by primary } \\
\text { care }\end{array}$ & $93.6 \%(44)$ & $87.7 \%(50)$ \\
\hline
\end{tabular}

ASCVD, atherosclerotic cardiovascular disease; BC, breast cancer, $\mathrm{SD}$, standard deviation.

Cancer survivors seen within 3 months were matched with controls based on age and medical comorbidity of hypertension, hyperlipidemia, and diabetes mellitus.

in individuals with a diagnosis of hypertension, no significant differences were observed in the percentage of individuals with controlled blood pressure (Figure 1B). In individuals with an indication for statin therapy, cancer survivors were more likely to have a current prescription for statin therapy $(P<.05)$ (Figure 1C). Multivariate analysis for statin therapy (Nagelkerke R-squared value was 0.08 for the model) showed that BC survivors were more likely to be on statin therapy $(P=.001)$. For lipid screening the Nagelkerke R-squared value was 0.06 . Cancer survivors were more likely to receive lipid screening within 3 years $(P=.017)$.

\section{Cardiovascular Screening and Lipid Management in BC Survivors by Race}

African American BC survivors were found to have no differences in cardiovascular screening, blood pressure control and statin use as compared with White BC survivors (Figure 2A). African American $\mathrm{BC}$ survivors were less likely to have a body weight in the appropriate range (Figure $2 \mathrm{~A}, P<.01$ ). African American women were noted to have a higher incidence of hypertension (Figure 2B). African American $\mathrm{BC}$ survivors did not have a higher incidence of diabetes than White BC survivors in our study (35\% vs $20 \%, P=.09$ ).

\section{Older BC Survivors Have Increased Cholesterol Screening}

Older BC survivors have increased cholesterol screening as compared with those 40 to 55 years old $(P<.001)$ (Figure 3A). Individuals aged 66 to 75 years old had an increased incidence of hypertension and diabetes compared with younger BC survivors (Figure 3B).

\section{Discussion}

Cardiovascular morbidity and mortality disproportionately impact BC survivors compared with women who do not have a personal history of the disease, in part due to previous cancer treatments received. CVD is often a late effect of treatment, with the onset of this disease often occurring several years after primary cancer treatment has been completed. In this article, we characterized the CVD screening practices among BC survivors who had an established primary care provider. Our study demonstrates that BC survivors received improved cardiovascular screening and statin therapy when appropriate as compared with peers. Primary care predominantly provides cardiovascular screening and management in this population with the majority of patients seeing primary care more recently than cancer specialists.

Our study demonstrates increased use of cholesterol screening and statins in cancer survivors as compared with matched controls. Blood pressure 
Figure 1. Cardiovascular screening and statin therapy in breast cancer $(\mathrm{BC})$ survivors. A, BC survivors are more likely to have received cholesterol screening and statin therapy than matched controls. B, Subgroup analysis of BC survivors with a diagnosis of hypertension have no differences in blood pressure control compared with comorbidity matched peers. C, Subgroup analysis of BC survivors are more likely than matched controls to have an active statin prescription if indicated. Sample size is indicated in parentheses for each group. Abbreviation: BMI, body mass index. *P $<.05, * * P<.01, * * * P<.001$.

A

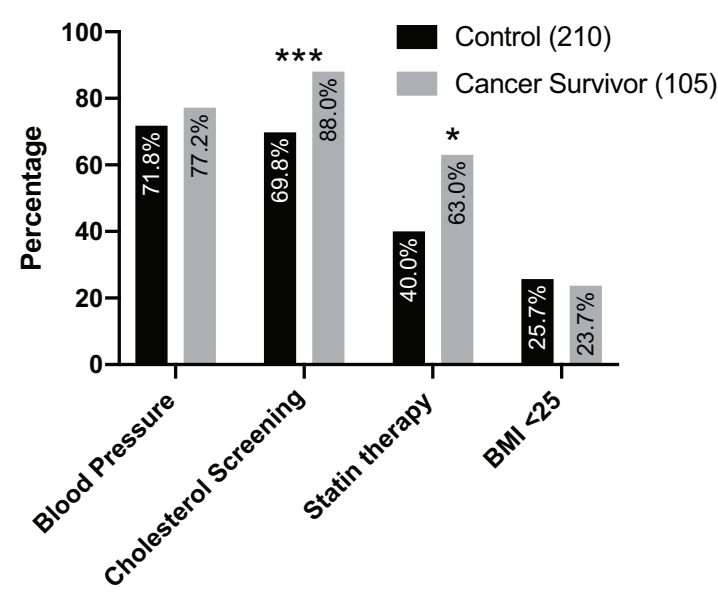

B Blood Pressure $<140 / 90$ with
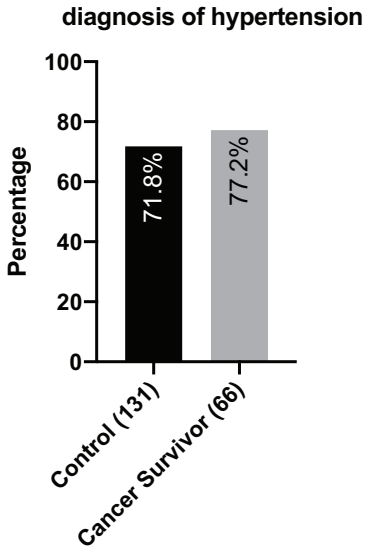

C Statin therapy if indicated

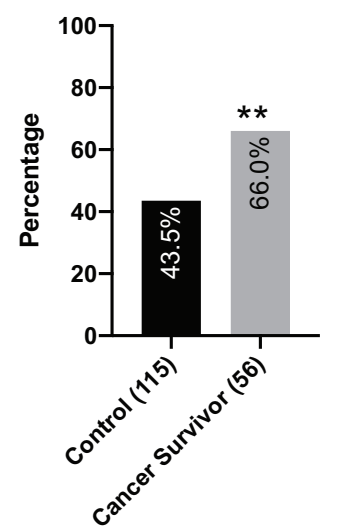

control for individuals with a diagnosis of hypertension did not differ between cancer survivors and controls. Based on the time of data extraction, guidelines from the Eighth Joint National Committee for blood pressure control were utilized in this study. ${ }^{44}$ The finding of comparable blood pressure control in cancer survivors is a significant finding given that endocrine therapies, antiangiogenic therapies, and other cancer therapies have been associated with elevated blood pressures. ${ }^{45-47}$

Figure 2. Cardiovascular screening, management, and comorbidities in African American and White breast cancer (BC) survivors. A, No differences in cardiovascular screening and management were observed between White and African American BC survivors; however, African American BC survivors were found to have a decreased incidence of having a body mass index less than $25 \mathrm{~kg} / \mathrm{m} 2$. B, African American BC survivors are more likely to have a diagnosis of hypertension. Sample size is indicated in parentheses for each group. Abbreviation: BMI, body mass index. $* \boldsymbol{P}<.05, * * * \boldsymbol{P}<.001$.

A

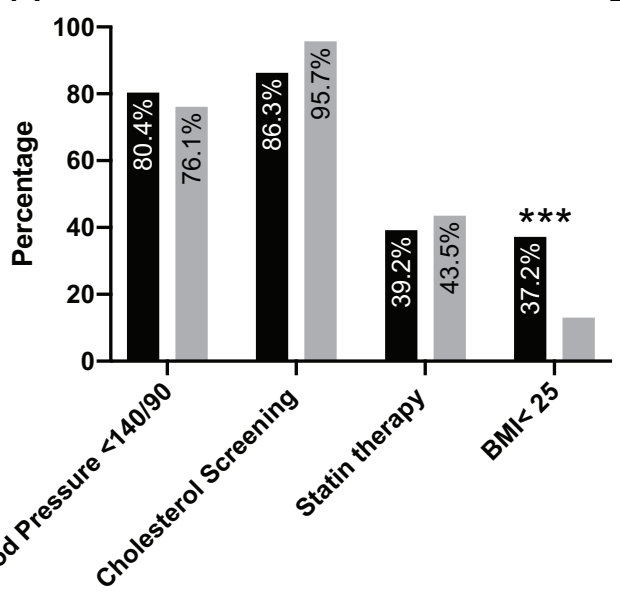

B

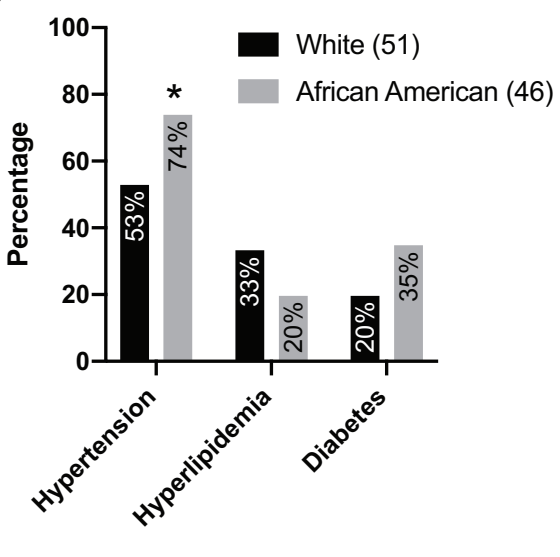


Figure 3. Impact of age on cardiovascular screening breast cancer (BC) survivors. A, Cardiovascular screening and management of $\mathrm{BC}$ survivors stratified by age. Older individuals are more likely to have received cholesterol screening than younger counterparts. B, Incidence of comorbidities based on age in BC survivors. Older BC survivors have a higher incidence of hypertension. Sample size is indicated in parentheses for each group. $* P<.05$, $* * \boldsymbol{P}<.01, * * * \boldsymbol{P}<.001$.

A

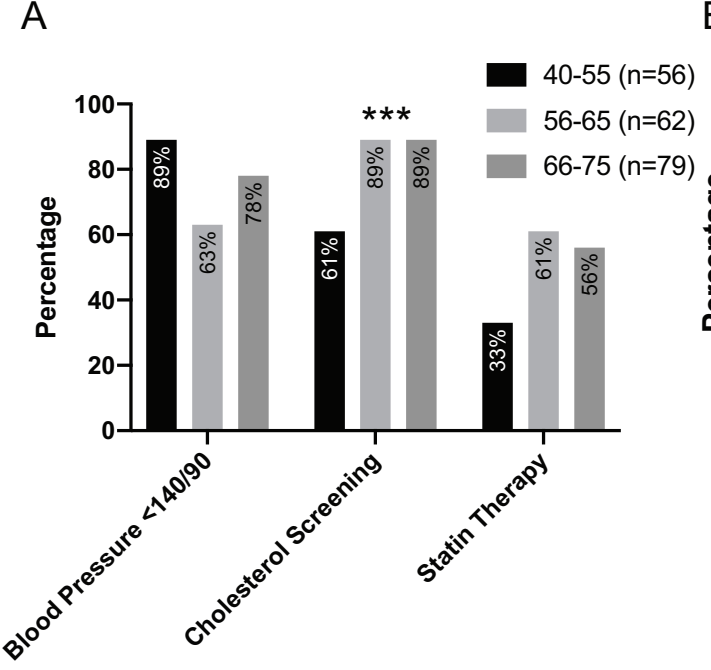

B

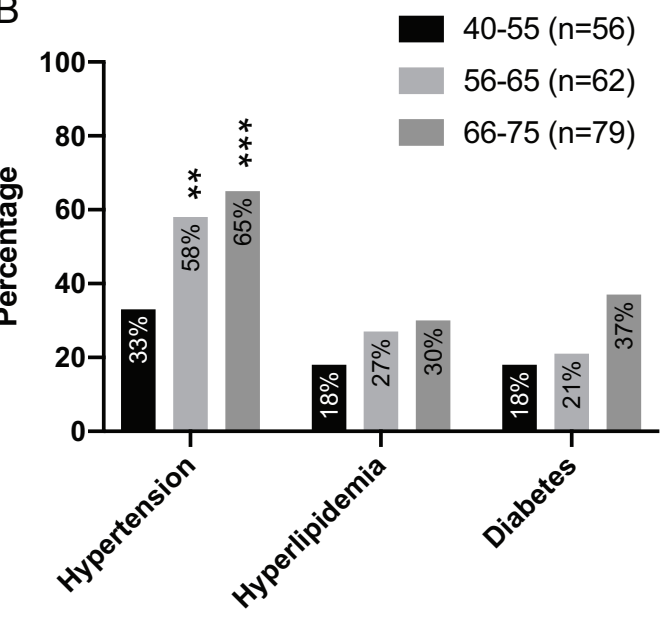

Previous studies regarding the receipt of preventive health screenings and in particular cholesterol screening have been limited and have had conflicting results. Synder et al conducted a longitudinal study and multi-cohort study evaluating cholesterol screening and other preventive health services in Medicare recipients compared with matched controls using claims-based data and found decreased rates of screening in cancer survivors compared with controls except when a comorbidity-based cohort analysis was conducted. ${ }^{48-50}$ Other studies focused on Medicare BC survivors and cancer survivors using both claims-based and interview data have demonstrated increased preventive health services including cholesterol screening. ${ }^{16,39}$ When individual cancers types have been studied, there is variation in the receipt of recommended acute and chronic care that cancer survivors receive. ${ }^{50}$ In these studies, the frequency of cholesterol screening was suboptimal. When we examine a population of patients with an identified primary care provider with associated recent visit to their primary care provider, cholesterol screening was increased in BC survivors compared with controls. Based on our findings, we concluded that BC survivors with an identified primary care provider are more likely to received cholesterol screening and statin treatment. Although not directly evaluated in this study, we believe that this is a result of both patient and provider factors. BC survivors may be more likely to pursue recommended preventive health given previous history of surviving cancer diagnosis as a focus on improved ongoing health. Health care providers may be more likely to conduct cholesterol screening in $\mathrm{BC}$ survivors due to the increasing awareness of their cardiovascular risk. It would be beneficial for future qualitative work to examine the factors that contribute to this increased screening on a patient, provider, and system level.

African American women have a lower incidence of BC compared with White women; however, African American women have a higher mortality when diagnosed with $\mathrm{BC}$ even when matched with White women with the same stage at time of diagnosis. ${ }^{51,52}$ As BC treatment has improved and cancer survivors are having improved treatment outcomes, this has disproportionally benefited White women. In this study, we found that there were no differences between African American women and White women regarding screening. African American BC survivors were more likely to have a diagnosis of hypertension than White BC survivors, but this was consistent with the incidence in the general population.

The current study has some advantages compared with previously conducted studies. In addition to focusing on cholesterol screening, we have 
further included a cardiovascular risk evaluation and inclusion of statin therapy when appropriate based on risk, major cardiac event, or diagnosis of diabetes. For this reason, we have focused on a younger population of $\mathrm{BC}$ survivors with average age of 63 years compared with 75 years for previous studies. ${ }^{16,48,50}$ Although the benefit of statin use for primary prevention for individuals at high cardiovascular risk has been established, insufficient evidence is available for its use in individuals over the age of 75 years. ${ }^{53}$ In addition, in comparison with previously conducted studies, this study has a higher percentage of African American women. More than $42 \%$ of our sample were African American whereas these women made up 10\% or less of the samples examined in previous reports. ${ }^{16,54}$

The current study has several limitations. Although the primary care practices included employed both internal and family medicine physicians and advanced practice providers, the study population was a population of BC survivors seen at a single academic institution. To further evaluate screening practices in those with a usual source of care, individuals with a primary care provider were selected for inclusion and as such the data cannot be extrapolated to those without a primary care provider. The screening rates reported are likely to be higher than for a cohort of all BC survivors. Our data are limited by information that can be extracted from the electronic health record and does not include screening or management obtained from other sources. Data including dietary habits, physical activity, aspirin use, and socioeconomic status could not be included.

BC survivors are at increased risk of CVD, for which screening and management is conducted predominantly in the primary care setting. The results of this study demonstrate that BC survivors are more likely than matched controls to be screened for CVD risk. Future research may benefit from investigation as to whether development of cardiovascular risk assessment and management guidelines that consider an individual's cancer diagnosis and treatment history would improve cardiovascular outcomes for BC survivors. As majority of cardiovascular screening and management is conducted in the primary care setting, communication between cancer specialists and primary care regarding an individual's treatment history and associated cardiovascular risk is essential to the care of cancer survivors.

\footnotetext{
To see this article online, please go to: http://jabfm.org/content/ 33/6/894.full.
}

\section{References}

1. Miller KD, Siegel RL, Lin CC, et al. Cancer treatment and survivorship statistics, 2016. CA Cancer J Clin 2016;66:271-89.

2. Maas AH, Ottevanger $\mathrm{N}$, Atsma $\mathrm{F}$, Cramer MJ, Leiner T, Poortmans P. Cardiovascular surveillance in breast cancer treatment: a more individualized approach is needed. Maturitas 2016;89:58-62.

3. Montazeri K, Unitt C, Foody JM, Harris JR, Partridge AH, Moslehi J. ABCDE steps to prevent heart disease in breast cancer survivors. Circulation 2014;130:e157-e159.

4. Ording AG, Boffetta P, Garne JP, Witt Nyström PM, Cronin-Fenton D, Frøslev T, et al. Relative mortality rates from incident chronic diseases among breast cancer survivors-A 14 year follow-up of fiveyear survivors diagnosed in Denmark between 1994 and 2007. Eur J Cancer 2015;51:767-75.

5. Jordan JH, Thwin SS, Lash TL, Buist DSM, Field TS, Haque R, et al. Incident comorbidities and allcause mortality among 5 -year survivors of Stage I and II breast cancer diagnosed at age 65 or older: a prospective-matched cohort study. Breast Cancer Res Treat 2014;146:401-9.

6. Jones DH, Nestore M, Henophy S, Cousin J, Comtois AS. Increased cardiovascular risk factors in breast cancer survivors identified by routine measurements of body composition, resting heart rate and arterial blood pressure. Springerplus 2014;3:150.

7. Jones LM, Stoner L, Brown C, Baldi C, McLaren B. Cardiovascular disease among breast cancer survivors: the call for a clinical vascular health toolbox. Breast Cancer Res Treat 2013;142:645-53.

8. Patnaik JL, Byers T, DiGuiseppi C, Dabelea D, Denberg TD. Cardiovascular disease competes with breast cancer as the leading cause of death for older females diagnosed with breast cancer: a retrospective cohort study. Breast Cancer Res 2011;13:R64.

9. Patnaik JL, Byers T, Diguiseppi C, Denberg TD, Dabelea $\mathrm{D}$. The influence of comorbidities on overall survival among older women diagnosed with breast cancer. J Natl Cancer Inst 2011;103:1101-11.

10. Bradshaw PT, Stevens J, Khankari N, Teitelbaum SL, Neugut AI, Gammon MD. Cardiovascular disease mortality among breast cancer survivors. Epidemiology 2016;27:6-13.

11. Strongman H, Gadd S, Matthews A, et al. Medium and long-term risks of specific cardiovascular diseases in survivors of 20 adult cancers: a populationbased cohort study using multiple linked UK electronic health records databases. Lancet 2019;21:10411054.

12. Barac A, Murtagh G, Carver JR, Chen MH, Freeman AM, Herrmann J, et al. Cardiovascular health of patients with cancer and cancer survivors: a roadmap to the next level. J Am Coll Cardiol 2015;65:2739-46. 
13. Koene RJ, Prizment AE, Blaes A, Konety SH. Shared risk factors in cardiovascular disease and cancer. Circulation 2016;133:1104-14.

14. Wolf I, Sadetzki S, Catane R, Karasik A, Kaufman B. Diabetes mellitus and breast cancer. Lancet Oncol 2005;6:103-11.

15. Han H, Guo W, Shi W, Yu Y, Zhang Y, Ye X, et al. Hypertension and breast cancer risk: a systematic review and meta-analysis. Sci Rep 2017;7:44877.

16. Lowenstein LM, Ouellet JA, Dale W, Fan L, Gupta Mohile S. Preventive care in older cancer survivors. J Geriatr Oncol 2015;6:85-92.

17. Lenihan DJ, Cardinale DM. Late cardiac effects of cancer treatment. J Clin Oncol 2012;30:3657-64.

18. Hooning MJ, Botma A, Aleman BM, Baaijens MHA, Bartelink H, Klijn JGM, et al. Long-term risk of cardiovascular disease in 10-year survivors of breast cancer. J Natl Cancer Inst 2007;99:365-75.

19. Baxi SS, Pinheiro LC, Patil SM, Pfister DG, Oeffinger KC, Elkin EB. Causes of death in longterm survivors of head and neck cancer. Cancer 2014;120:1507-13.

20. Zagar TM, Cardinale DM, Marks LB. Breast cancer therapy-associated cardiovascular disease. Nat Rev Clin Oncol 2016;13:172-84.

21. Daher IN, Daigle TR, Bhatia N, Durand JB. The prevention of cardiovascular disease in cancer survivors. Tex Heart Inst J 2012;39:190-8.

22. Jones LW, Haykowsky MJ, Swartz JJ, Douglas PS, Mackey JR. Early breast cancer therapy and cardiovascular injury. J Am Coll Cardiol 2007;50:143541.

23. Nathan PC, Amir E, Abdel-Qadir H. Cardiac outcomes in survivors of pediatric and adult cancers. Can J Cardiol 2016;32:871-80.

24. Kirkham AA, Virani SA, Campbell KL. The utility of cardiac stress testing for detection of cardiovascular disease in breast cancer survivors: a systematic review. Int J Womens Health 2015;7:127-40.

25. Chen J, Lu Y, Zheng Y. Incidence and risk of hypertension with bevacizumab in non-small-cell lung cancer patients: a meta-analysis of randomized controlled trials. Drug Des Devel Ther 2015;9:475160.

26. Chen CL. Cardiovascular prevention in the cancer survivor. Curr Atheroscler Rep 2015;17:484484.

27. Bhatt DL. Ask the doctor. I had radiation and chemotherapy treatments for breast cancer. My cancer doctor sent me to a cardiologist for an ultrasound. Are there cardiac risks to cancer treatments? Harv Heart Lett 2014;25:2.

28. Kenyon M, Mayer DK, Owens AK. Late and longterm effects of breast cancer treatment and surveillance management for the general practitioner. J Obstet Gynecol Neonatal Nurs 2014;43:382-98.

29. Dossett LA, Hudson JN, Morris AM, et al. The primary care provider (PCP)-cancer specialist relationship: a systematic review and mixed-methods meta-synthesis. CA Cancer J Clin 2017;67:156-69.

30. Cheung WY, Neville BA, Cameron DB, Cook EF, Earle CC. Comparisons. of patient and physician expectations for cancer survivorship care. J Clin Oncol 2009;27:2489-95.

31. Klabunde CN, Ambs A, Keating NL, Doucette WR, Tisnado D, Clauser S, et al. The role of primary care physicians in cancer care. J Gen Intern Med 2009;24:1029-36.

32. Mayer DK, Nasso SF, Earp JA. Defining cancer survivors, their needs, and perspectives on survivorship health care in the USA. Lancet Oncol 2017;18:e11-e8.

33. Lawrence RA, McLoone JK, Wakefield CE, Cohn RJ. Primary care physicians' perspectives of their role in cancer care: a systematic review. J Gen Intern Med 2016;31:1222-36.

34. Nekhlyudov L. "Doc, should I see you or my oncologist?": a primary care perspective on opportunities and challenges in providing comprehensive care for cancer survivors. J Clin Oncol 2009;27:2424-6.

35. Nekhlyudov L. Integrating primary care in cancer survivorship programs: models of care for a growing patient population. Oncologist 2014;19:579-82.

36. Rubin G, Berendsen A, Crawford SM, et al. The expanding role of primary care in cancer control. Lancet Oncol 2015;16:1231-72.

37. Mandelzweig L, Chetrit A, Amitai T, Silverman B, Siegelmann-Danieli N, Sadetzki S. Primary prevention and screening practices among long-term breast cancer survivors. Cancer Causes Control 2017;28:657-66.

38. Snyder CF, Frick KD, Herbert RJ, Blackford AL, Neville BA, Lemke KW, et al. Comorbid condition care quality in cancer survivors: role of primary care and specialty providers and care coordination. J Cancer Surviv 2015;9:641-9.

39. Earle CC, Burstein HJ, Winer EP, Weeks JC. Quality of non-breast cancer health maintenance among elderly breast cancer survivors. J Clin Oncol 2003;21:1447-51.

40. Chidwick K, Strongman H, Matthews A, Stanway S, Lyon AR, Smeeth L, et al. Statin use in cancer survivors versus the general population: cohort study using primary care data from the UK clinical practice research datalink. BMC Cancer 2018;18:1018.

41. Lewallen S, Courtright P. Epidemiology in practice: case-control studies. Community Eye Health 1998;11:57-8.

42. James PA, Oparil S, Carter BL, et al. 2014 evidence-based guideline for the management of high blood pressure in adults: report from the panel members appointed to the Eighth Joint National Committee (JNC 8). JAMA 2014;311:507-20.

43. Stone NJ, Robinson JG, Lichtenstein A. The new cholesterol treatment guidelines. $\mathrm{N}$ Engl J Med 2014;370:1957. 
44. Armstrong C. JNC8 guidelines for the management of hypertension in adults. Am Fam Physician 2014;90:503-4.

45. Mehta LS, Watson KE, Barac A, Beckie TM, Bittner V, Cruz-Flores S, et al. Cardiovascular disease and breast cancer: where these entities intersect: a scientific statement from the American Heart Association. Circulation 2018;137:e30-e66.

46. Yeo W, Mo FKF, Pang E, Suen JJS, Koh J, Loong $\mathrm{HHF}$, et al. Profiles of lipids, blood pressure and weight changes among premenopausal Chinese breast cancer patients after adjuvant chemotherapy. BMC Womens Health 2017;17:55.

47. Blaes A, Beckwith H, Florea N, Hebbel R, Solovey A, Potter D, et al. Vascular function in breast cancer survivors on aromatase inhibitors: a pilot study. Breast Cancer Res Treat 2017;166:541-7.

48. Snyder CF, Frick KD, Kantsiper ME, Peairs KS, Herbert RJ, Blackford AL, et al. Prevention, screening, and surveillance care for breast cancer survivors compared with controls: changes from 1998 to 2002. J Clin Oncol 2009;27:1054-61.

49. Snyder CF, Frick KD, Peairs KS, Kantsiper ME, Herbert RJ, Blackford AL, et al. Comparing care for breast cancer survivors to non-cancer controls: a five-year longitudinal study. J Gen Intern Med 2009;24:469-74.

50. Snyder CF, Frick KD, Herbert RJ, Blackford AL, Neville BA, Wolff AC, et al. Quality of care for comorbid conditions during the transition to survivorship: differences between cancer survivors and noncancer controls. J Clin Oncol 2013;31:1140-8.

51. Yedjou CG, Tchounwou PB, Payton M, Fonseca DD, Lowe L, Alo RA. Assessing the Racial and Ethnic Disparities in Breast Cancer Mortality in the United States. Int J Environ Res Public Health 2017;14:486.

52. Tangka FKL, Subramanian S. Importance of implementation economics for program planning-evaluation of CDC's colorectal cancer control program. Eval Program Plann 2017;62:64-6.

53. Bibbins-Domingo K, Grossman DC, Curry SJ, Jr, Davidson KW, Epling JW, Jr, García FAR, et al. Statin use for the primary prevention of cardiovascular disease in adults: US Preventive Services Task Force recommendation statement. JAMA 2016;316:1997-2007.

54. Stewart JH, Bertoni AG, Staten JL, Levine EA, Gross CP. Participation in surgical oncology clinical trials: gender-, race/ethnicity-, and age-based disparities. Ann Surg Oncol 2007;14:3328-34. 